\title{
1 Exosome-Mediated mRNA Delivery For SARS-CoV-2 Vaccination
}

2

$3 \quad{ }^{1}$ Shang Jui Tsai, ${ }^{1}$ Chenxu Guo, ${ }^{2}$ Alanna Sedgwick, ${ }^{2}$ Saravana Kanagavelu, ${ }^{2}$ Justin Nice,

$4 \quad{ }^{2}$ Sanjana Shetty, ${ }^{2}$ Connie Landaverde, ${ }^{2}$ Nadia A. Atai, and ${ }^{1 *}$ Stephen J. Gould

$6{ }^{1}$ Department of Biological Chemistry, The Johns Hopkins University School of Medicine,

7725 North Wolfe Street, Baltimore, MD, 21205

$8 \quad{ }^{2}$ Capricor Therapeutics, Inc. 8840 Wilshire Blvd. 2nd Floor, Beverly Hills, CA 90211

10 Correspondence:

11 Name: Stephen J. Gould

12 Address: $\quad 725$ North Wolfe Street

16 Phone number: 4438479918

17 Email: $\quad$ sgould@jhmi.edu

19 Running title: Exosomal SARS-CoV-2 vaccine

21 Key Words: COVID19, spike, nucleocapsid, exosomes, mRNA, lipid, antibody, T-cell,

22 extracellular vesicles 


\section{Abstract}

26 Expression-dependent, Spike-only vaccines have been developed, deployed, and shown

27 to be effective in the fight against SARS-CoV-2. However, additional approaches to

28 vaccine development may be needed to meet existing and future challenges posed by

29 emerging Spike variant strains, as well as a likely need for different antigen-delivery

30 systems that are safe and effective for regular, periodic re-administration. We report here

31 the development of mRNA-loaded exosomes, demonstrate that they can mediate the

32 functional expression of heterologous proteins in vitro and in vivo, and have fewer

33 adverse effects than comparable doses of lipid nanoparticles. Furthermore, we applied

34 this approach to the development of an exosome-based, multiplexed mRNA vaccine that

35 drives expression of immunogenic SARS-CoV-2 Nucleocapsid and Spike proteins. This

36 vaccine elicited long-lasting cellular and humoral responses to Nucleocapsid and to

37 Spike, demonstrating that exosome-based mRNA formulations represent a previously

38 unexplored platform in the fight against COVID-19 and other infectious diseases. 


\section{Introduction}

42 Severe acute respiratory syndrome coronavirus 2 (SARS-CoV-2) is the causative agent

43 of COVID-19 1,2. COVID-19 typically presents with symptoms common to many

44 respiratory infections, including fever and cough, but in many cases progresses to more

45 severe disease that may include acute respiratory distress, disseminated disease, and

46 death ${ }^{34-8}$. SARS-CoV-2 entered the human population in late 2019 as the result of a

47 zoonotic leap and is most closely related to coronaviruses endemic to bats (Chiroptera)

489 . SARS-CoV-2 is the third recent zoonotic betacoronavirus to enter the human

49 population, the others being responsible for the outbreaks of severe acute respiratory

50 syndrome (SARS-CoV) in $2002^{10}$ and middle east respiratory syndrome (MERS-CoV) in

512012 (Memish et al., 2013), indicative of a generally susceptibility of human populations

52 to coronavirus zoonoses. These zoonoses are more distantly to human endemic

53 betacoronaviruses (OC43, HKU1, etc.) that also cause respiratory infections of milder

54 effect ${ }^{11}$ ). While SARS-CoV-2 infection is associated with lower mortality than SARS-CoV

55 or MERS-CoV, SARS-CoV-2 initially displayed a higher rate of transmission, quickly

56 became a major cause of morbidity and mortality worldwide

57 (https://www.cdc.gov/coronavirus/2019-ncov/hcp/clinical-guidance-management-

58 patients.html)(coronavirus.jhu.edu), and has continued to evolve into numerous variant

59 strains that display even more-elevated rates of transmission and an emerging resistance

60 to antibody-based neutralization ${ }^{12-15}$.

61

62 SARS-CoV-2 enters host cells via a multistep pathway that begins with binding between

63 the Spike protein on the virus surface and its cognate receptor proteins on the host cell 
64 surface. These include angiotensin-converting enzyme II (ACE2) 1,16,17, neuropilin-1 18,19,

65 and perhaps also CD147 ${ }^{20}$. Following virus-cell binding, host cell proteases (e.g.

66 TMPRSS2 ${ }^{16}$, cathepsins ${ }^{21}$, etc.) cleave Spike, potentiating Spike-catalyzed fusion

67 between the viral and cellular membranes and functional infection of the host cell. Not

68 surprisingly, SARS-CoV-2 receptors and proteases are expressed within the respiratory

69 tract, consistent with its respiratory mode of transmission ${ }^{22}$. However, they are also

70 expressed in many other cell types, allowing SARS-CoV-2 to spread within the body and

71 impact multiple organ systems (brain, heart, gastrointestinal tract, circulatory system,

72 immune system, etc. $\left.{ }^{18,19,23-26}\right)$.

74 Following virus-cell membrane fusion, the viral genomic RNA (gRNA) is translated to 75 generate two large polyproteins, open reading frame 1 (orf1a) and orf1ab, which are 76 processed to release 16 nonstructural proteins (nsp1-16) ${ }^{27}$. These early proteins prime

77 the host cell for virus replication and mediate the synthesis of subgenomic viral RNAs and

78 their unique protein products. These include a dozen or more additional proteins,

79 including the SARS-CoV-2 structural proteins Nucleocapsid (N), Spike (S), Membrane

$80(\mathrm{M})$, and Envelope (E). Spike, Membrane and Envelope are integral membrane proteins,

81 co-translationally translocated into the endoplasmic reticulum (ER), that subsequently

82 drive virion formation while also incorporating the Nucleocapsid and its bound gRNA as

83 well as some other ancillary proteins ${ }^{28,29}$, with virus release via lysosomal exocytosis

84 (Ghosh et al., 2020) []. The released viral particles are $100 \mathrm{~nm}$ diameter, display

85 prominent Spike protrusions from the cell surface and a lumen containing Nucleocapsid-

86 gRNA complexes ${ }^{30}$. SARS-CoV-2 biogenesis also involves extensive processing of its 
87 Spike protein at a polybasic site, generating S1 and S2 forms of Spike, with the N-

88 terminal, receptor-binding S1 fragment bound non-covalently to the fusogenic,

89 membrane-anchored S2 fragment ${ }^{16,31,32}$.

90

91 Vaccine design should mirror, and ideally improve on, the correlates of protective

92 immunity that arise from natural infections. It is now well-established that SARS-CoV-2

93 infection generates potent cellular and humoral immune responses to viral proteins that

94 in most cases reverse the course of disease, clear the viral infection, and confer

95 resistance to reinfection in both people and in animal models ${ }^{33-3637-39}$. Disease-preventing

96 vaccines have previously been developed for animal coronaviruses ${ }^{40}$ and have been

97 successfully developed and deployed for SARS-CoV-2 ${ }^{41-47}$, a development that is likely

98 to save millions of lives. However, these first-generation SARS-CoV-2 vaccines only elicit

99 immunity to a single viral protein, Spike, the rapid evolution of which may impair vaccine

100 efficacy ${ }^{12-15}$. Furthermore, the Spike-only vaccine approach ignores the fact that a

101 primary correlate of immunity in COVID-19 patients is the array of potent immune

102 reactions to the SARS-CoV-2 Nucleocapsid protein ${ }^{48}$.

103

104 Here we describe an expression-dependent SARS-CoV-2 vaccine that combines 105 exosome-based delivery, multiplexed mRNA formulation, induction of immunity to both 106 Spike and Nucleocapsid, and antigen design that involves expressing Nucleocapsid in a 107 form designed for improved antigen presentation. Exosomes are small extracellular 108 vesicles (sEVs) of $\sim 30-150 \mathrm{~nm}$ in diameter that are made by all cells, abundant in all 109 biofluids, and mediate intercellular transmission of signals and macromolecules, including 
bioRxiv preprint doi: https://doi.org/101101/202011.06.371419; this version posted March 8, 2021. The copyright holder for this preprint

110 RNAs ${ }^{49}$. Allogenic exosome transplantations and transfusions have been practices in

111 one form or another for more than a century and have never been associated with any

112 adverse effects. Moreover, exosomes have already been shown effective for delivery of

113 RNA-based therapeutics ${ }^{50,51}$. The remainder of this report describes the production of

114 engineered exosome/mRNA formulations, their ability to drive protein expression in

115 cultured cells and animals, their improved safety relative to LNPs, and their use as a

116 multiplexed, exosome-based SARS-CoV-2 vaccine that elicited immunity to multiple viral

117 antigens, including Nucleocapsid as well as Spike. 


\section{Results}

120 Exosomes display robust ability to deliver functional mRNAs in vitro and in vivo

121 Exosomes are capable of delivering functional RNAs to target cells ${ }^{50,51}$, but so too are 122 synthetic lipid vesicles, often referred to as lipid nanoparticles (LNPs) ${ }^{52}$. To better 123 understand the dynamics of mRNA delivery by these two natural and synthetic forms of 124 soluble vesicles, we generated matched formulations of mRNA-loaded exosomes and 125 mRNA-loaded LNPs. Exosomes were purified from the culture of 293F cells (Fig. 1), 126 LNPs ${ }^{52}$ were obtained from a commercial provider, and equal amounts of each (by 127 vesicle number) were loaded with a synthetic mRNA encoding the hybrid 128 luciferase/fluorescent protein Antares2 (Antares2 is comprised of the luciferase teLuc 129 fused to two copies of the fluorescent protein CyOFP1 (CyOFP1-teLuc-CyOFP1), emits far-red shift light via bioluminescent resonance energy transfer ${ }^{53}$ ). Equal amounts of these

131 matched exo-mRNA and LNP-mRNA formulations were then incubated at low and high 132 doses with human cells, followed by an overnight incubation to allow for Antares2 protein 133 expression. The next day, the cells were incubated with diphenylterazine (DTZ), a cell134 permeable substrate (luciferin) for Antares2, and assayed for DTZ-dependent, Antares2catalyzed light emission (Fig. 2). At low-dose administration, Antares2 expression was $13625 \%$ higher in cells treated with the exo-mRNA formulation than with the LNP-mRNA 137 formulation ( $\mathrm{n}=6, p=0.0016)$. The difference in Antares2 expression was even more 138 pronounced at high-dose administration, as the exo-mRNA-treated cells expressed far 139 more Antares2 activity than the LNP-exo-treated cells (16-fold; $\mathrm{n}=6 ; p=0.00035)$. 
141 This large difference in particle-mediated Antares2 expression was caused by a drop in

142 LNP-mRNA-mediated expression, raising the possibility that LNP administration is

143 inhibitory at high levels of administration. This in turn raised the possibility of general

144 toxicity of LNP administration, which we addressed by following the short-term

145 consequences of exosome and LNP injections in mice. Animals were injected (i.m.) with

146 equal numbers of either exosomes or LNPs $(50 \mathrm{ml}$ of ??? particles $/ \mathrm{ml})$, returned to their

147 cages for three days, and then sacrificed and processed for organ histology by an

148 independent testing laboratory (Fig. 3A). No abnormalities were detected in control 149 animals (5/5) or in animals injected with exosomes (5/5). In contrast, only one of the LNP-

150 injected animals (1/5) displayed normal spleen histology, as 4/5 animals showed an

151 increase in red pulp. Adverse LNP effects may also explain the $\sim 5 \%$ reduction in body

152 mass $(\mathrm{n}=5 ; p=0.05)$ we observed at 3 days post-injection (Fig. $3 \boldsymbol{B})$.

154 The robust expression of exosome-delivered mRNA in vitro and the absence of exosome-

155 associated adverse effects led us to next test whether RNA-loaded exosomes might also

156 be able to drive Antares2 expression in vivo. Towards this end, we injected adult mice

157 (0.05 ml volume, intramuscular (i.m.) administration) with Antares2 mRNA-loaded

158 exosomes, returned the animals to cages to allow for Antares2 expression. 24 hours later,

159 the control (uninjected) and treated mice were injected (i.p.) with a solution of the

160 Antares2 luciferin DTZ and imaged immediately using a real-time bioluminescent imaging

161 (BLI) system to visualize exosome-mediated, mRNA-directed Antares2 expression.

162 Control animals displayed no significant light emission upon DTZ injections whereas

163 animals that had been injected with the mRNA-loaded exosome formulation displayed 
164 robust light emission (Fig. 3). These observations demonstrate that RNA-loaded

165

166

167

168

169

170

171 mRNAs, one of which expresses the form of SARS-CoV-2 Spike $\left(\mathrm{S}^{\mathrm{W} 1}\right)$ encoded by the

172 initial viral isolate ${ }^{1}$. The second mRNA expresses a fusion protein (LSNME) comprised

173 of the SARS-CoV-2 Nucleocapsid protein, as well as fragments of the Spike, Membrane,

174 and Envelope proteins, all inserted in the extracellular domain of human Lamp1 (this

175 Lamp1-based fusion protein aims to induce anti-SARS-CoV-2 immunity by targeting viral

176 protein fragments to the $\mathrm{MHC}$ Class I and II antigen presentation pathways ${ }^{54,5556}$ ).

177 Transfection of these mRNAs into HEK293 cells (Fig. 4) resulted in expression of Spike

178 at the cell surface but also at internal organelles (shown elsewhere to be lysosomes ${ }^{57}$ ),

179 whereas expression of LSNME led to its accumulation in what appears to the 180 181

182

183 A single exosome-mRNA formulation containing both the LSNME and $\mathrm{S}^{\mathrm{W} 1}$ mRNAs 184 (hereafter referred to as the LSNME/SW1 vaccine) was injected (i.m.) into 13 weeks-old 185 male C57BL/6J mice (Fig. 5). The vaccine was dosed at 4 ug or 0.25 ug equivalents of 186 each mRNA and injections were performed on day 1 (primary immunization), day 21 (1st 
187 boost), and day 42 (2nd boost). Blood $(0.1 \mathrm{~mL})$ was collected on days 14, 35, 56, 70 and

84. On day 84 the animals were sacrificed to obtain tissue samples for histological

194 antibody production was expected in the case of the $\mathrm{N}$ protein, as the LSNME mRNA is 195 designed to stimulate cellular immune responses rather than the production of anti-N 196 antibodies.

\section{LSNME/S ${ }^{W 1}$ vaccination induces cellular immune responses to $\mathrm{N}$ and $\mathrm{S}$}

199 Vaccinated and control animals were also interrogated for the presence of antigen200 reactive CD4+ and CD8+ T-cells. This was carried out by collecting splenocytes at the 201 completion of the trial (day 84) using a CFSE proliferation assay in the presence or 202 absence of recombinant $\mathrm{N}$ and $\mathrm{S}$ proteins. These experiments revealed that vaccination

203 had induced a significant increase in the percentages of CD4 ${ }^{+}$T-cells and CD8 ${ }^{+}$T-cells

204 that proliferated in response to addition of either recombinant $\mathrm{N}$ protein or recombinant S 205 protein to the culture media (Fig. 6A-D). These vaccine-specific, antigen-induced 206 proliferative responses demonstrate that the LSNME/SW1 vaccine achieved its primary 207 goal, which was to prime the cellular arm of the immune system to generate N-reactive $208 \mathrm{CD}^{+}$and $\mathrm{CD}^{+}$T-cells, and also S-reactive $\mathrm{CD}^{+}$and $\mathrm{CD}^{+}{ }^{+}$T-cells. In additional 209 experiments, we stained antigen-induced T-cells cells for the expression of interferon 
210 gamma (IFN $\gamma$ ) and interleukin 4 (IL4). These experiments revealed that the S-reactive

$211 \mathrm{CD}^{+}$T-cell population displayed elevated expression of the Th1-associated cytokine

212 IFN $\gamma$, and to a lesser extent, the Th2-associated cytokine IL4 (Fig 7). In contrast, N-

213 reactive T-cells failed to display an $\mathrm{N}$-induced expression of either IFN $\gamma$ or IL4.

214

\section{Absence of vaccine-induced adverse reactions}

216 Control and vaccinated animals were examined regularly for overall appearance, general

217 behavior, and injection site inflammation (redness, swelling). No vaccine-related

218 differences were observed in any of these variables, and animals from all groups

219 displayed similar age-related increases in body mass (supplemental figure 1).

220 Vaccination also had no discernable effect on blood cell counts (supplemental figure 2).

221 Histological analyses were performed on all animals at the conclusion of the study by an

222 independent histology service, which reported that vaccinated animals showed no

223 difference in overall appearance of any of the tissues that were examined. Representative

224 images are presented for brain, lung, heart, liver, spleen, kidney, and side of injection

225 skeletal muscle in an animal from each of the trial groups (Fig. 8). 


\section{Discussion}

229 Exosomes are natural products of human cells that are more 'self' than 'non-self'. Immune

systems are tolerant of the high levels of exosomes that are continuously present in all biofluids (e.g. blood, lymph, cerebrospinal fluid, vitreous, interstitial fluids, etc.) ${ }^{49,58}$.

232 Furthermore, there is no evidence of adverse effects of allogeneic exosome transfer,

233 whether of purified exosomes (from amniotic fluid, blood, etc.) or of inadvertent exosome

234 transfer during tissue transplantation, blood transfusion, plasma injection, etc. In this

235 context, the fact that exosomes normally participate in pathways of vesicle-mediated, 236 intercellular RNA traffic ${ }^{59-61}$ indicates that exosomes may be an ideal vehicle for clinical

237 RNA delivery. The data presented here support this hypothesis by showing that that 238 exosome-mRNA formulations can support the in vivo, functional expression of proteins 239 as diverse as soluble cytoplasmic enzymes, viral structural proteins, and synthetic fusion 240 proteins.

241

242 Our findings are also relevant to the ongoing battle against SARS-CoV-2. Current vaccine 243 strategies are all centered on inducing immunity to Spike, but Spike-only vaccines are 244 susceptible to escape effects whenever and antigenically shifted Spike variants starts to 245 spread in susceptible populations. While we are developing strategies designed to 246 address this challenge by improved design of expression-dependent Spike vaccines, we 247 are also working to address it by generating a multiplexed mRNA vaccine that delivers 248 two or more mRNAs, one encoding Spike and the others encoding Nucleocapsid and 249 perhaps fragments of other proteins as well. One limitation of this approach is that 250 Nucleocapsid is a cytoplasmic protein rather than a surface antigen, a topology that limits 
251 its efficacy in vaccination studies. However, this limitation can be overcome by expressing

252 Nucleocapsid as part of a fusion with the lysosomal resident protein Lamp1, which places

253 Nucleocapsid protein in the correct compartments for Class I and Class II antigen

254 presentation (ER and lysosome/MHC Class II compartment, respectively). This approach

255 was realized in our LSNME/SW1 vaccine, which elicited strong cellular immune responses

256 to Nucleocapsid as well as to Spike. Vaccinated animals displayed antigen-induced CD4 ${ }^{+}$

257 and $\mathrm{CD}^{+}$T-cell responses to both Nucleocapsid and to Spike that persisted for nearly

258 two months after immunization. Furthermore, when these cell populations were

259 interrogated for antigen-induced expression of the cytokines IFN $\gamma$ and IL4, we detected

260 elevated expression of IFN $\gamma$ in CD4+ T-cells exposed to exogenous Spike protein, as well

261 as a more modest Spike-induced expression of IL4. These results raise the possibility

262 that the exosome-based LSNME/SW1 vaccine induces the kind of Th1-skewed cellular

263 immune response desired for an anti-viral vaccine. Vaccinated animals also developed

264 durable antibody responses to the Nucleocapsid and the Spike proteins that were

265 sustained at relatively constant levels over the 7 weeks following immunization. This

266 multi-antigen immune response bodes well for this approach in the next generation of

267 SARS-CoV-2 vaccines that will be needed to protect against the emerging array of

268 antigenically distinct SARS-CoV-2 viral strains and their ever-increasing spectrum of

269 Spike protein mutations.

270

271 In conclusion, the results presented in this study validate the use of multiplexed exosome-

272 mRNA formulations for functional delivery of mRNAs both in cultured cells and in live

273 animals. The successful use of exosomes to deliver Antares2 mRNA opens the door to 
274 follow-on studies aimed at optimizing exosome-RNA formulation conditions, as well as

275 for characterizing the time-dependence of Antares2 expression, biodistribution of

276 exosome-mediated RNA expression, injection site effects, and exosome-mediated tissue

277 tropism. As for the future development of exosome-based SARS-CoV-2 mRNA vaccines,

278 we anticipate that follow-on studies will demonstrate multiple advantages of exosome-

279 based delivery, improved antigen designed, and most importantly, improved protective

280 effects that arise from immunization with multiple viral antigens, and particularly

281 Nucleocapsid, which is a main target of anti-SARS-CoV-2 immunity in COVID-19 patients

$282{ }^{48}$ and has proven effective in vaccine studies of other coronaviruses ${ }^{54}$. Furthermore, the

283 fact that exosomes can be deployed at high concentrations without adverse effects on

284 cells or animals bodes well for their future use in dosing regimens that require higher-

285 level or ongoing repeated injections. 


\section{Methods}

Cell culture

291 293F cells (Gibco, Cat.\# 51-0029) were tested for pathogens and found to be free of viral

292 (cytomegalovirus, human immunodeficiency virus I and II, Epstein Barr virus, hepatitis B 293 virus, and parvovirus B19), and bacterial (Mycoplasma) contaminants. Cells were 294 maintained in FreeStyle 293 Expression Medium (Gibco, \#12338-018) and incubated at $29537^{\circ} \mathrm{C}$ in $8 \% \mathrm{CO}_{2}$. For exosome production, 293F cells were seeded at a density of $1.5 \mathrm{x}$ $29610^{\wedge} 6 \mathrm{cell} / \mathrm{ml}$ in shaker flasks in a volume of $\sim 1 / 4$ the flask volume and grown at a shaking 297 speed of 110 rpm. HEK293 cells were grown in Dulbecco's modified Eagle's medium 298 supplemented with $10 \%$ fetal calf serum.

300 Exosome purification

$301293 F$ cells were grown in shaker cultures for a period of three days. Cells and large cell 302 debris were removed by centrifugation at $300 \times g$ for 5 minutes followed by $3000 \times g$ for 30315 minutes. The resulting supernatant was passed through a $0.22 \mu \mathrm{m}$ sterile filtration filter 304 unit (Thermo Fisher, \#566-0020) to generate a clarified tissue culture supernatant 305 (CTCS). The CTCS was concentrated by centrifugal filtration (Centricon Plus-70, Ultracel306 PL Membrane, 100 kDa size exclusion, Millipore Sigma \# UFC710008), with 120 mLs 307 CTCS concentrated to $\sim 0.5 \mathrm{mLs}$. Concentrated CTCS was then purified by size exclusion 308 chromatography (SEC) in 1x PBS (qEV original columns/35 nm: Izon Science, \#SP5), 309 with the exosomes present in each $0.5 \mathrm{~mL}$ starting sample eluting in three $0.5 \mathrm{~mL}$ 310 fractions. Purified exosomes were reconcentrated using Amicon ${ }^{\circledR}$ Ultra-4 100 kDa cutoff 
311 spin columns (\#UFC810024). This process yielded a population of exosomes/small EVs

312 that have the expected ultrastructure and size distribution profile of human exosomes and

313 contain the exosomal marker proteins CD9 and CD63 (Fig. 8), at a concentrating effect

314 of $\sim 500$-fold, to a final concentration of $\sim 2 \times 10^{12}$ exosomes $/ \mathrm{ml}$, representing an average 315 recovery of $35 \%$.

317 Nanoparticle Tracking Analysis (NTA)

318 Vesicle concentrations and size distribution profiles of exosome preparations were 319 measured by nanoparticle tracking analysis (NTA) using a NanoSight NS300 (Malvern

320 Panalytical, United Kingdom) in 1x PBS clarified by filtration through a $0.22 \mu \mathrm{m}$ sterile

321 filtration unit. Measurements were carried out in triplicates at ambient temperature with

322 fixed camera settings (level of 14 , screen gain of 10 , detection threshold 3 , and 323 temperature of $21.7-22.2^{\circ} \mathrm{C}$ ). Immunostaining nanoparticle tracking analysis (NTA) was 324 performed using fluorescently labeled antibody conjugate directed against human CD63 325 (AlexaFluor488-conjugated clone 460305; R\&D Systems (Minneapolis, USA)). The 326 fluorescently labeled anti-CD63-antibody $(1 \mu \mathrm{l})$ was incubated with exosomes $(9 \mu \mathrm{l})$ for 2

327 hours at room temperature in the dark, then diluted by addition of $1 \mathrm{ml}$ of sterile-filtered 328 PBS (Thermo Fisher, USA) and examined for exosome abundance, size, and CD63 329 immunoreactivity using a Particle Metrix ZetaView ${ }^{\circledR}$ TWIN device. Samples were 330 visualized in scatter mode using the $488 \mathrm{~nm}$ laser and standard instrument settings 331 (sensitivity: 80, shutter: 100, min. brightness: 30; min. area: 10; max. area: 1000) in 332 fluorescence mode with standard fluorescence settings (sensitivity: 88 , shutter: 100, min. 
333 brightness: 25; min. area: 10; max. area: 1000). The resulting videos were analysed with

334 the ZetaView® software 8.05.10 (Particle Metrix, Germany).

336 Immunoblots

337 Exosome and cell lysates were separated by SDS-PAGE using pre-cast, 4-15\% gradient 338 gels (Bio-Rad 4561086) and transferred to PVDF membranes (ThermoFisher, \#88518).

339 Membranes were blocked, probed with antibodies directed against CD9 (clone HI9a;

340 BioLegend), CD63 (MX-49.129.5), CD81 (555675; BD Pharmingen), or HSP90 (sc341 13119; Santa Cruz Biotechnology), then washed, exposed to HRP-conjugates of goat 342 secondary antibodies (Jackson Immunoresearch), washed, and processed for 343 chemiluminescent imaging using HRP-activated chemiluminescence detection solution 344 (Amersham ECL Western Blotting Detection Reagents; cat\# RPN2106), and imaged 345 using a GE Amersham Imager 600. Images were exported as JPEG files, analyzed using 346 ImageJ software, and processed using Photoshop (Adobe).

348 Electron Microscopy and light microscopy

349 Exosomes were fixed by addition of formaldehyde to a final concentration of $4 \%$. Carbon350 coated grids were placed on top of a drop of the exosome suspension. Next, grids were 351 placed directly on top of a drop of $2 \%$ uranyl acetate. The resulting samples were 352 examined with a Tecnai-12 G2 Spirit Biotwin transmission electron microscope (John 353 Hopkins University, USA). Fluorescence micrographs of Antares2 expression in 354 transfected HEK293 cells were captured as PNG files using an EVOS M7000 microscope 355 equipped with an Olympus UPlanSAPo 40x/0.95 objective. 
Production of mRNA-loaded exosomes and LNPs

358 mRNAs were obtained from a commercial provider (Trilink). mRNAs were purified using

359 RNeasy columns (Qiagen) and resuspended in DNase-free, RNase-free water using

360 nuclease-free tips and tubes. Purified mRNAs were pre-incubated with a coating of

361 polycationic lipids and then mixed with equal amounts of either purified exosomes or

362 LNPs (DOTAP/DOPE, \#F50102, FormuMAx Scientific Inc) at $4^{\circ} \mathrm{C}$ for 10 minutes.

363 Formulations were either used immediately or frozen at $-80^{\circ} \mathrm{C}$ and thawed rapidly prior to 364 use.

Luciferase measurements and bioluminescent imaging

367 HEK293 cells were incubated with exosome-mRNA formulations overnight under 368 standard culture conditions. Antares2 luciferase activity was measured by Live cell 369 bioluminescence was collected after incubating with substrate diphenylterazine (MCE, $370 \mathrm{HY}-111382$ ) at final concentration of $50 \mu \mathrm{M}$ for 3 minutes. Readings were collected using 371 a SpectraMax i3x (Molecular Devices). For in vivo studies, thirteen months-old, female

372 Balb/c mice (Jackson Laboratory) housed under pathogen-free conditions at the Cedars-

373 Sinai Medical Center animal facility were used to study the expression of Exosome-

374 Anteres2 mRNA expression 24 hours after injection. Intramuscular injections were at a 375 volume of $50 \mu \mathrm{ls}$ per mouse containing $5 \mathrm{ug}$ mRNA. After 24 hours the animals were 376 imaged using an IVIS Spectrum imager (PerkinElmer, Waltham, MA) (All animal 377 experimentation was performed following institutional guidelines for animal care and were 378 approved by the Cedars-Sinai Medical Center IACUC (\#8602). 
Animal experimentation

381 All animal experimentation was performed following institutional guidelines for animal 382 care and were approved by the Cedars-Sinai Medical Center IACUC (\#8602). All 383 injections were at a volume of $50 \mu \mathrm{ls}$. Experiments involved injection of exosomes, LNPS, 384 and Antares2 mRNA-loaded exosomes were performed with BALB/c mice (Jackson 385 Laboratory). Immunization with mRNA-loaded exosomes were performed on thirteen 386 weeks-old, male C57BL/6J mice (Jackson Laboratory) housed under pathogen-free 387 conditions at the Cedars-Sinai Medical Center animal facility. Blood $(\sim 0.1 \mathrm{~mL})$ was 388 collected periodically from the orbital vein. At day 84 , mice were deeply anesthetized using isoflurane, euthanized by cervical dislocation, and processed using standard 390 surgical procedures to obtain spleen, lung, brain, heart, liver, kidney, muscle, and other 391 tissues. Spleens were processed for splenocyte analysis, and all tissues were processed 392 for histological analysis by fixation in $10 \%$ neutral buffered formalin. Histological analysis 393 was performed by the service arm of the HIC/Comparative Pathology Program of the 394 University of Washington.

397 Mouse IgG antibody production against SARS-CoV-2 antigens was measured by 398 enzyme-linked immunosorbent assays (ELISA). For antigens S1 (RBD) and N, pre399 coated ELISA plates from RayBiotech were utilized (IEQ-CoV S RBD-IgG; IEQ-CoVN$400 \mathrm{lg}$ ), and the experiments were performed according to the manufacturer's instructions, 401 with modification. Briefly, mouse plasmas at dilutions of 1:50 were added to antigen pre- 
402 coated wells in duplicates and incubated at room temperature (RT) for 2 hours on a

403 shaker (200 rpm). The plates were washed 4 times with wash buffer followed by blocking

404 for 2 hours at RT with 1\% BSA in PBS. Mouse antibodies bound to the antigens coated

405 on the ELISA plates were detected using HRP-conjugated goat anti-mouse secondary

406 antibodies (Jackson Immuno Research Inc.) Plates were washed 4 times with washing

407 buffer, and developed using TMB substrate (RayBiotech). Microplate Reader was used

408 to measure the absorbance at $650 \mathrm{~nm}$ (SpectraMaxID3, Molecular Devices, with SoftMax

409 Pro7 software).

410

411 Single cell splenocyte preparation

412 After terminal blood collection, mice were euthanized, and part of fresh spleens were

413 harvested. Single cell splenocyte preparation was obtained by machinal passage through

414 a $40 \mu \mathrm{m}$ nylon cell strainer (BD Falcon, \#352340). Erythrocytes were depleted using

415 Ammonium-Chloride-Potassium (ACK) lysis buffer (Gibco, \#A10492-01), and

416 splenocytes were washed using R10 media by centrifuging at 300x $g$ for 5 minutes at RT.

417 R10 media (RPMI 1640 media (ATCC, Cat\#302001) supplemented with 10\% fetal bovine

418 serum (FBS) (Atlas, \#E01C17A1), 50 M 2-mercaptoethanol (Gibco, \#21985-023),

419 penicillin/streptomycin (VWR life sciences, \#K952), and 10 mM HEPES (Gibco, \#15630-

420 080)) was used for all analyses of blood cells. The cells were resuspended in fresh media

421 and counted in hemocytometer counting chamber to be used in subsequent experiments.

422

423 Spleen lymphocyte population characterization 
424 Splenocytes $\left(2 \times 10^{5}\right.$ cells/mouse) were resuspended in $100 \mu \mathrm{L}$ of $10 \%$ FBS in $1 \times$ PBS 425 and incubated with fluorochrome-conjugated antibodies for surface staining of CD3

426 (Invitrogen, \#17-0032-82) CD4 (Biolegend, \#100433), CD8 (Biolegend, \#100708), B220

427 (BD, \#552771) CD11c (Invitrogen, \#17-0114-81), F4/80 (Invitrogen, \#MF48004) Ly6G

428 (Invitrogen, \#11-9668-80) and Ly6C (BD, \#560592)) for 30 minutes at $4{ }^{\circ} \mathrm{C}$ in the dark.

429 Following incubation, samples were washed twice with $200 \mu \mathrm{Ls} 10 \%$ FBS in 1x PBS and

430 centrifuged at $300 \times g$ for 5 minutes at RT to remove unbound antibodies. Next the cells

431 were fixed with $100 \mu \mathrm{Ls}$ ICS fixation buffer (Invitrogen, \#00-8222-49). Samples were 432 analyzed on a FACS Canto II (BD Biosciences) with 2,000 - 10,000 recorded 433 lymphocytes. The data analysis was performed using FlowJo 10 software (FlowJo, LLC) 434 and presented as a percentage change in the immune cell population compared to the 435 vehicle-treated group.

437 SARS-CoV-2 antigen-specific T cell proliferation assay using CFSE

438 Splenocytes were resuspended at $10^{6}$ cells/mL in $10 \%$ FBS in 1xPBS and stained with 439 carboxyfluorescein succinimidyl ester (CFSE) (Invitrogen, \#C34554) by rapidly mixing 440 equal volume of cell suspension with $10 \mu \mathrm{M}$ CFSE in 10\% FBS in 1x PBS for 5 minutes 441 at $37^{\circ} \mathrm{C}$. The labeled cells were washed three times with R10 complete medium. The cells 442 were incubated for 96 hours in the presence of $10 \mu \mathrm{g} / \mathrm{mL}$ SARS-CoV-2 antigens $\mathrm{N}$ or S1 443 (Acro Biosystems, \#NUN-C5227; SIN-C52H4) or medium alone as negative control. After 44496 hours, cells were washed with $200 \mu \mathrm{Ls} 10 \%$ FBS in $1 \times$ PBS and centrifuged at $300 \times g$ 445 for 5 minutes at RT. Cells were then stained with anti-CD3-APC (Invitrogen, \#17-0032446 82), anti-CD4-PerCP-Cy5.5 (Biolegend, \#100433), and anti-CD8-PE antibodies 
447 (Biolegend, \#MCD0801) for 30 minutes at $4^{\circ} \mathrm{C}$. The stained cells were washed twice with $448200 \mu \mathrm{Ls}$ 1x PBS and analyzed on a FACS Canto II (BD Biosciences). For analysis,

449 lymphocytes were first gated for CD3+ T-cells, then for CD4+/CD8- or CD8+/CD4450 populations. The data analysis was performed using FlowJo 10 software (FlowJo LLC).

452 Intracellular staining for cytokines

$4532.0 \times 10^{5}$ splenocytes/mouse were incubated for 72 hours in the presence of $10 \mu \mathrm{g} / \mathrm{mL}$ 454 SARs-CoV2 antigens N or S1 (Acro Biosystems) or R10 medium alone (negative control). 455 After 72 hours, the cells were washed with fresh R10 medium and incubated with phorbol 456 myristate acetate (PMA) at concentration of $50 \mathrm{ng} / \mathrm{mL}$ (Sigma, \#P1585), ionomycin at 457 concentration of $350 \mathrm{ng} / \mathrm{mL}$ (Invitrogen, \#124222), and GogiPlug at concentration of 0.8 $458 \mu \mathrm{L} / \mathrm{mL}$ (Invitrogen, \#51-2301KZ) for 4 hours to amplify cytokine expression in T cells. The 459 cells were then washed with 10\% FBS in 1x PBS and stained with anti-CD3-APC, anti460 CD4-PerCP-Cy5.5, and anti-CD8-PE antibodies (Added above) for 30 minutes at $4^{\circ} \mathrm{C}$ in 461 dark. The cells were washed twice with 1xPBS followed by permeabilization step using 462 ready-to-use buffer (Invitrogen \#00-8333-56). Next the cells were fixed with ICS fixation 463 bufferAdded above for 10 minutes at RT in dark and stained intracellular for IFN-Y 464 (eBioscience, \#11-7311-82), IL-10 (eBioscience, \#11-7101-82), IL-4 (Invitrogen, \#12465 7041-41) and Foxp3 (Invitrogen, \#12-5773-80) overnight at $4^{\circ} \mathrm{C}$ in permeabilization buffer.

466 The stained cells were analyzed on a BD FACS Canto II with 5,000 - 10,000 recorded 467 lymphocytes. The data analysis was performed using FlowJo 10 software. 
bioRxiv preprint doi: https://doi org/101101/2020.11.06 371419; this version posted March 8, 2021. The copyright holder for this preprint (which was not certified by peer review) is the author/funder, who has granted bioRxiv a license to display the preprint in perpetuity. It is made available under aCC-BY-NC-ND 4.0 International license.

470 Statistical analysis was performed using GraphPad Prism 8 software for Windows/Mac

471 (GraphPad Software, La Jolla California USA) or Excel. Results are reported as mean \pm

472 standard deviation or mean \pm standard error, and the differences were analyzed using

473 Student's t-test or one-way analysis of variance.

474

475 
476 Sources of Support: This study was conducted with support from Capricor and from

477 Johns Hopkins University.

478

479 Disclosures: S.J.G is a paid consultant for Capricor, holds equity in Capricor, and is co-

480 inventor of intellectual property licensed by Capricor. S.J.T. is co-inventor of intellectual

481 property licensed by Capricor. C.G. is co-inventor of intellectual property licensed by

482 Capricor. A.S., S.K., J.N., S.S., C.L., and N.A. are employees of Capricor.

483

484 Acknowledgments: We thank Omid Sheikh for outstanding technical assistance during

485 the course of these studies.

486

487

488

489

490 


\section{$491 \quad$ Figure Legends}

492

493 Figure 1. Exosome purification and characterization. (A) Schematic of exosome

494 purification from cultures of $293 \mathrm{~F}$ cells grown in chemically defined media. (B) NTA

495 analysis of purified exosomes showed a mean exosome diameter of $\sim 115 \mathrm{~nm}$. (C)

496 Negative stain electron micrograph of purified exosomes. Bar, $100 \mathrm{~nm}$. (D)

497 Immunofluorescent NTA analysis of 293F-derived exosomes that had been labeled

498 previously using fluorescently labeled anti-CD63 antibody. (E) Immunoblot analysis of 499 equal proportions of $293 \mathrm{~F}$ cell and exosome lysates using antibodies specific for the 500 exosomal markers CD81, CD9, \& CD63, as well as the control cytoplasmic protein Hsp90.

501

502 Figure 2. Exosomes display superior mRNA delivery characteristics. Relative luciferase 503 activities (average +/- standard error of the mean) of cells treated with low or high 504 concentrations of mRNA-loaded exosomes or mRNA-loaded LNPs.

505

506 Figure 3. Effect of exosome and LNP injections on organ histology and body mass. (A)

507 H\&E staining of tissue sections from BALB/c mice that had been injected three days 508 earlier with $50 \mathrm{ml}$ of PBS, exosomes $\left(10^{12} / \mathrm{ml}\right)$, or LNPs $\left(10^{12} / \mathrm{ml}\right)$. (B) Body mass 509 measurements prior to and at 3 days after injection. All animals were subjected to analysis 510 by an independent pathology service, which noted spleen abnormalities in 4/5 LNP511 treated animals but no abnormalities in control or exosome-treated animals. 
513 Figure 4. Real-time imaging of exosome-mediated, functional mRNA delivery. Combined

514 bioluminescent and light images of control mice and treated mice immediately following

515 i.p. administration of DTZ. Treated mice had been injected with Antares2 mRNA-loaded

516 exosomes 24 hours prior to imaging. Radiance is in photons/second/area

$517\left(\mathrm{~cm}^{2}\right) /$ steradian.

518

519 Figure 5. Expression of $S^{W 1}$ and LSNME following mRNA transfection. (A, B)

520 Fluorescence micrographs of HEK293 cells stained with DAPI and a plasma from a

521 COVID-19 patient. (C-F) Fluorescence micrographs of HEK293 cells stained with DAPI

522 and plasmas from a COVID-19 patient following their transfection with the (C, D) SW1_

523 encoding mRNA and (E, F) the LSNME-encoding mRNA. Bar, $50 \mu \mathrm{m}$.

524

525 Figure 6. LSNME/S ${ }^{W 1}$ vaccination induces antibody responses to SARS-CoV-2 N and S

526 protein. (A) Schematic of immunization and blood/tissue collection timeline. (B) Anti-N

527 ELISA results of diluted plasma from (grey bars and black circles) individual six control

528 mice, (orange bars and black squares) six mice immunized with $0.25 \mu \mathrm{g}$ equivalents of

529 each mRNA, and (rust bars and black triangles) six mice immunized with $4 \mu \mathrm{g}$ equivalents

530 of each mRNA. (C) Anti-S1 ELISA results of diluted plasma from (grey bars and black

531 circles) individual six control mice, (orange bars and black squares) six mice immunized

532 with $0.25 \mu \mathrm{g}$ equivalents of each mRNA, and (rust bars and black triangles) six mice

533 immunized with $4 \mu \mathrm{g}$ equivalents of each mRNA. Height of bars represents the mean,

534 error bars represent +/- one standard error of the mean, and the statistical significance of 
535 differences between different groups is reflected in Student's t-test values of * for $<0.05$,

${ }^{* *}$ for $<0.005$, and *** for $<0.0005$.

538 Figure 7. LSNME/SW1 vaccination induces $C D 4^{+}$and $C D 8^{+} T$-cell responses. CFSE-

539 labeled splenocytes were interrogated by flow cytometry following incubation in the

540 absence or presence of $(A, B)$ purified, recombinant $N$ protein or $(C, D)$ purified,

541 recombinant $S$ protein, and for antibodies specific for CD4 and CD8. Differences in

542 proliferation of $\mathrm{CD}^{+}$cells and $\mathrm{CD}^{+}$cells were plotted for (grey bars and black circles)

543 individual six control mice, (orange bars and black squares) six mice immunized with 0.25

$544 \mu \mathrm{g}$ equivalents of each mRNA, and (rust bars and black triangles) six mice immunized

545 with $4 \mu \mathrm{g}$ equivalents of each mRNA. Height of bars represents the mean, error bars

546 represent +/- one standard error of the mean, and the statistical significance of differences

547 between different groups is reflected in Student's t-test values of * for $<0.05$ and ${ }^{* *}$ for

$548<0.005$

549

550 Figure 8. LSNME/S ${ }^{W 1}$ vaccination leads to S-induced expression of IFN $\gamma$ and IL4 by CD4 ${ }^{+}$

551 T-cells. Splenocytes were interrogated by flow cytometry following incubation in the

552 absence or presence of $(A, B)$ purified, recombinant $N$ protein or $(C, D)$ purified, 553 recombinant S protein, and labeling with antibodies specific for CD4 or CD8, and for IFN $\gamma$

554 or IL4. Differences in labeling for IFN $\gamma$ or IL4 in $\mathrm{CD}^{+} \mathrm{CD}^{+}$cell populations were plotted

555 for (grey bars and black circles) individual six control mice, (orange bars and black

556 squares) six mice immunized with $0.25 \mu \mathrm{g}$ equivalents of each mRNA, and (rust bars and

557 black triangles) six mice immunized with $4 \mu \mathrm{g}$ equivalents of each mRNA. Height of bars 
558 represents the mean, error bars represent +/- one standard error of the mean, and the

559 statistical significance of differences between different groups is reflected in Student's t-

560 test values of * for $<0.05$.

561

562 Figure 9. Absence of tissue pathology upon LSNME/SW1 vaccination. Representative

563 micrographs from histological analysis (hematoxylin and eosin stain) of lung, brain, heart,

564 liver, kidney, spleen, and muscle (side of injection) of animals from (upper row) control

565 mice, (middle row) mice immunized with the lower dose of the LSNME/SW1 vaccine, and

566 (lower row) mice immunized with the higher dose of the LSNME/SW1 vaccine.

567

568

569

570

571 
572 Supplemental Figure 1. Equivalent growth of vaccinated and control animals. Body mass

573 of all mice was measured over the course of the study and plotted as average $+/-$ the

574 standard error of the mean, relative to the body mass at the initiation of the trial, with

575 groups reported as (grey lines and circles) control mice, (orange lines and squares) lower

576 dose-treated mice, and (rust lines and triangles) higher dose-treated mice.

577

578 Supplemental Figure 2. Vaccination does not induce changes in the proportional

579 representation of key blood cell populations. Splenocytes were interrogated by flow

580 cytometry using antibodies specific for (A) B220, (B) Ly6C, (C) CD11c, and (D) CD3.

$581 \mathrm{CD}^{+}$cells were further differentiated by staining for (E) CD4 and (F) CD8. No statistically

582 significant differences were detected in these subpopulations of white blood cells.

583

584

585 


\section{References}

5881 Zhou, P. et al. A pneumonia outbreak associated with a new coronavirus of probable bat $589 \quad$ origin. Nature 579, 270-273, doi:10.1038/s41586-020-2012-7 (2020).

5902 Coronaviridae Study Group of the International Committee on Taxonomy of, V. The 591 species Severe acute respiratory syndrome-related coronavirus: classifying 2019-nCoV and naming it SARS-CoV-2. Nat Microbiol 5, 536-544, doi:10.1038/s41564-020-0695-z (2020).

5943 Gandhi, R. T., Lynch, J. B. \& Del Rio, C. Mild or Moderate Covid-19. N Engl J Med 383, 17571766, doi:10.1056/NEJMcp2009249 (2020).

5964 Wang, D. et al. Clinical Characteristics of 138 Hospitalized Patients With 2019 Novel Coronavirus-Infected Pneumonia in Wuhan, China. JAMA 323, 1061-1069, doi:10.1001/jama.2020.1585 (2020). Zhou, F. et al. Clinical course and risk factors for mortality of adult inpatients with COVID19 in Wuhan, China: a retrospective cohort study. Lancet 395, 1054-1062, doi:10.1016/S0140-6736(20)30566-3 (2020).

6026 Force, A. D. T. et al. Acute respiratory distress syndrome: the Berlin Definition. JAMA 307, 2526-2533, doi:10.1001/jama.2012.5669 (2012).

6047 Richardson, S. et al. Presenting Characteristics, Comorbidities, and Outcomes Among 5700 Patients Hospitalized With COVID-19 in the New York City Area. JAMA 323, 20522059, doi:10.1001/jama.2020.6775 (2020).

608

609 8 Guo, T. et al. Cardiovascular Implications of Fatal Outcomes of Patients With Coronavirus (2020).

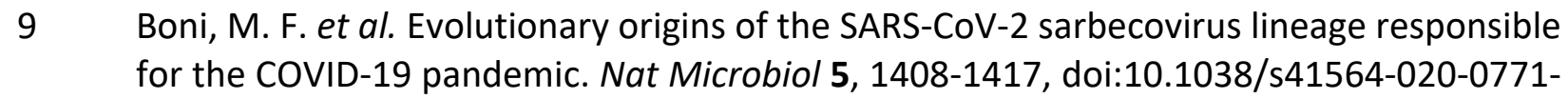
4 (2020).

11 Corman, V. M., Muth, D., Niemeyer, D. \& Drosten, C. Hosts and Sources of Endemic Human Coronaviruses. Adv Virus Res 100, 163-188, doi:10.1016/bs.aivir.2018.01.001 (2018).

12 Korber, B. et al. Tracking Changes in SARS-CoV-2 Spike: Evidence that D614G Increases Infectivity of the COVID-19 Virus. Cell 182, 812-827 e819, doi:10.1016/j.cell.2020.06.043 (2020).

62213 Alam, l. et al. CovMT: an interactive SARS-CoV-2 mutation tracker, with a focus on critical

623 variants. Lancet Infect Dis, doi:10.1016/S1473-3099(21)00078-5 (2021).

62414 Ho, D. et al. Increased Resistance of SARS-CoV-2 Variants B.1.351 and B.1.1.7 to Antibody 625 Neutralization. Res Sq, doi:10.21203/rs.3.rs-155394/v1 (2021). 
62615 Diamond, M. et al. SARS-CoV-2 variants show resistance to neutralization by many monoclonal and serum-derived polyclonal antibodies. Res Sq, doi:10.21203/rs.3.rs228079/v1 (2021).

62916 Hoffmann, M. et al. SARS-CoV-2 Cell Entry Depends on ACE2 and TMPRSS2 and Is Blocked 630 by a Clinically Proven Protease Inhibitor. Cell 181, 271-280 e278, doi:10.1016/j.cell.2020.02.052 (2020).

63217 Matheson, N. J. \& Lehner, P. J. How does SARS-CoV-2 cause COVID-19? Science 369, 510511, doi:10.1126/science.abc6156 (2020).

63418 Daly, J. L. et al. Neuropilin-1 is a host factor for SARS-CoV-2 infection. Science, doi:10.1126/science.abd3072 (2020).

19 Cantuti-Castelvetri, L. et al. Neuropilin-1 facilitates SARS-CoV-2 cell entry and infectivity. Science, doi:10.1126/science.abd2985 (2020).

20 Wang, K. et al. CD147-spike protein is a novel route for SARS-CoV-2 infection to host cells. Signal Transduct Target Ther 5, 283, doi:10.1038/s41392-020-00426-x (2020).

64021 Wei, J. et al. Genome-wide CRISPR Screens Reveal Host Factors Critical for SARS-CoV-2 Infection. Cell 184, 76-91 e13, doi:10.1016/j.cell.2020.10.028 (2021).

64222 Mason, R. J. Thoughts on the alveolar phase of COVID-19. Am J Physiol Lung Cell Mol Physiol 319, L115-L120, doi:10.1152/ajplung.00126.2020 (2020).

23 Nicin, L. et al. Cell type-specific expression of the putative SARS-CoV-2 receptor ACE2 in human hearts. Eur Heart J 41, 1804-1806, doi:10.1093/eurheartj/ehaa311 (2020).

24 Li, M. Y., Li, L., Zhang, Y. \& Wang, X. S. Expression of the SARS-CoV-2 cell receptor gene ACE2 in a wide variety of human tissues. Infect Dis Poverty 9, 45, doi:10.1186/s40249020-00662-x (2020).

25 Ziegler, C. G. K. et al. SARS-CoV-2 Receptor ACE2 Is an Interferon-Stimulated Gene in Human Airway Epithelial Cells and Is Detected in Specific Cell Subsets across Tissues. Cell 181, 1016-1035 e1019, doi:10.1016/j.cell.2020.04.035 (2020). Coronavirus Entry Factors. Cell Rep 32, 108175, doi:10.1016/j.celrep.2020.108175 (2020). V'Kovski, P., Kratzel, A., Steiner, S., Stalder, H. \& Thiel, V. Coronavirus biology and replication: implications for SARS-CoV-2. Nat Rev Microbiol, doi:10.1038/s41579-02000468-6 (2020).

28 Ujike, M. \& Taguchi, F. Incorporation of spike and membrane glycoproteins into coronavirus virions. Viruses 7, 1700-1725, doi:10.3390/v7041700 (2015).

29 Ruch, T. R. \& Machamer, C. E. The coronavirus E protein: assembly and beyond. Viruses 4, 363-382, doi:10.3390/v4030363 (2012).

662

30 Yao, H. et al. Molecular Architecture of the SARS-CoV-2 Virus. Cell 183, 730-738 e713, doi:10.1016/j.cell.2020.09.018 (2020).

31 Hoffmann, M., Kleine-Weber, H. \& Pohlmann, S. A Multibasic Cleavage Site in the Spike Protein of SARS-CoV-2 Is Essential for Infection of Human Lung Cells. Mol Cell 78, 779-784 Glycoprotein. Cell 181, 281-292 e286, doi:10.1016/j.cell.2020.02.058 (2020). macaques. Science 369, 818-823, doi:10.1126/science.abc5343 (2020). 
67034 Chandrashekar, A. et al. SARS-CoV-2 infection protects against rechallenge in rhesus 671 macaques. Science 369, 812-817, doi:10.1126/science.abc4776 (2020).

67235 Shan, C. et al. Infection with novel coronavirus (SARS-CoV-2) causes pneumonia in Rhesus

673 macaques. Cell Res 30, 670-677, doi:10.1038/s41422-020-0364-z (2020). Bosco-Lauth, A. M. et al. Experimental infection of domestic dogs and cats with SARS-CoV2: Pathogenesis, transmission, and response to reexposure in cats. Proc Natl Acad Sci U S A 117, 26382-26388, doi:10.1073/pnas.2013102117 (2020).

676

37 St John, A. L. \& Rathore, A. P. S. Early Insights into Immune Responses during COVID-19. J Immunol 205, 555-564, doi:10.4049/jimmunol.2000526 (2020).

679 applications to phase 3 vaccine candidates. Lancet, doi:10.1016/S0140-6736(20)32137-1 (2020).

Zost, S. J. et al. Potently neutralizing and protective human antibodies against SARS-CoV2. Nature 584, 443-449, doi:10.1038/s41586-020-2548-6 (2020).

689

690 Tizard, I. R. Vaccination against coronaviruses in

691

Walsh, E. E. et al. Safety and Immunogenicity of Two RNA-Based Covid-19 Vaccine Candidates. N Engl J Med, doi:10.1056/NEJMoa2027906 (2020).

693

694

695

Keech, C. et al. Phase 1-2 Trial of a SARS-CoV-2 Recombinant Spike Protein Nanoparticle Vaccine. N Engl J Med, doi:10.1056/NEJMoa2026920 (2020). Med, doi:10.1056/NEJMoa2022483 (2020). Respiratory Tracts against SARS-CoV-2. Cell 183, 169-184 e113, doi:10.1016/j.cell.2020.08.026 (2020).

45 Zhu, F. C. et al. Immunogenicity and safety of a recombinant adenovirus type-5-vectored COVID-19 vaccine in healthy adults aged 18 years or older: a randomised, double-blind, placebo-controlled, phase 2 trial. Lancet 396, 479-488, doi:10.1016/S01406736(20)31605-6 (2020).

700

701

702

47 Graham, S. P. et al. Evaluation of the immunogenicity of prime-boost vaccination with the

Bos, R. et al. Ad26 vector-based COVID-19 vaccine encoding a prefusion-stabilized SARSCoV-2 Spike immunogen induces potent humoral and cellular immune responses. NPJ Vaccines 5, 91, doi:10.1038/s41541-020-00243-x (2020).

709 replication-deficient viral vectored COVID-19 vaccine candidate ChAdOx1 nCoV-19. NPJ Vaccines 5, 69, doi:10.1038/s41541-020-00221-3 (2020).

48 Burbelo, P. D. et al. Sensitivity in Detection of Antibodies to Nucleocapsid and Spike Proteins of Severe Acute Respiratory Syndrome Coronavirus 2 in Patients With Coronavirus Disease 2019. J Infect Dis 222, 206-213, doi:10.1093/infdis/jiaa273 (2020).

49 Pegtel, D. M. \& Gould, S. J. Exosomes. Annu Rev Biochem 88, 487-514, doi:10.1146/annurev-biochem-013118-111902 (2019).

50 Kamerkar, S. et al. Exosomes facilitate therapeutic targeting of oncogenic KRAS in pancreatic cancer. Nature 546, 498-503, doi:10.1038/nature22341 (2017). 
71251 Li, L. et al. Extracellular vesicles carry microRNA-195 to intrahepatic cholangiocarcinoma 713 and improve survival in a rat model. Hepatology 65, 501-514, doi:10.1002/hep.28735 (2017).

$71552 \quad$ Hassett, K. J. et al. Optimization of Lipid Nanoparticles for Intramuscular Administration of mRNA Vaccines. Mol Ther Nucleic Acids 15, 1-11, doi:10.1016/j.omtn.2019.01.013 (2019).

53 Yeh, H. W. et al. Red-shifted luciferase-luciferin pairs for enhanced bioluminescence imaging. Nat Methods 14, 971-974, doi:10.1038/nmeth.4400 (2017).

54 Gupta, V. et al. SARS coronavirus nucleocapsid immunodominant T-cell epitope cluster is common to both exogenous recombinant and endogenous DNA-encoded immunogens. Virology 347, 127-139, doi:10.1016/j.virol.2005.11.042 (2006).

55 Vyas, J. M., Van der Veen, A. G. \& Ploegh, H. L. The known unknowns of antigen processing and presentation. Nat Rev Immunol 8, 607-618, doi:10.1038/nri2368 (2008).

56 Imai, J., Otani, M. \& Sakai, T. Distinct Subcellular Compartments of Dendritic Cells Used for Cross-Presentation. Int J Mol Sci 20, doi:10.3390/ijms20225606 (2019).

57 Guo, C. et al. The D614G Mutation Enhances the Lysosomal Trafficking of SARS-CoV-2 Spike. bioRxiv, doi:10.1101/2020.12.08.417022 (2020).

$58 \mathrm{Yu}, \mathrm{W}$. et al. Exosome-based Liquid Biopsies in Cancer: Opportunities and Challenges. Ann Oncol, doi:10.1016/j.annonc.2021.01.074 (2021).

73159 Skog, J. et al. Glioblastoma microvesicles transport RNA and proteins that promote tumour growth and provide diagnostic biomarkers. Nat Cell Biol 10, 1470-1476, doi:10.1038/ncb1800 (2008).

73460 Ratajczak, J. et al. Embryonic stem cell-derived microvesicles reprogram hematopoietic progenitors: evidence for horizontal transfer of mRNA and protein delivery. Leukemia 20, 847-856, doi:10.1038/sj.leu.2404132 (2006). extracellular vesicles in mammalian cells and its applications. Nat Rev Mol Cell Biol 21, 585-606, doi:10.1038/s41580-020-0251-y (2020). 


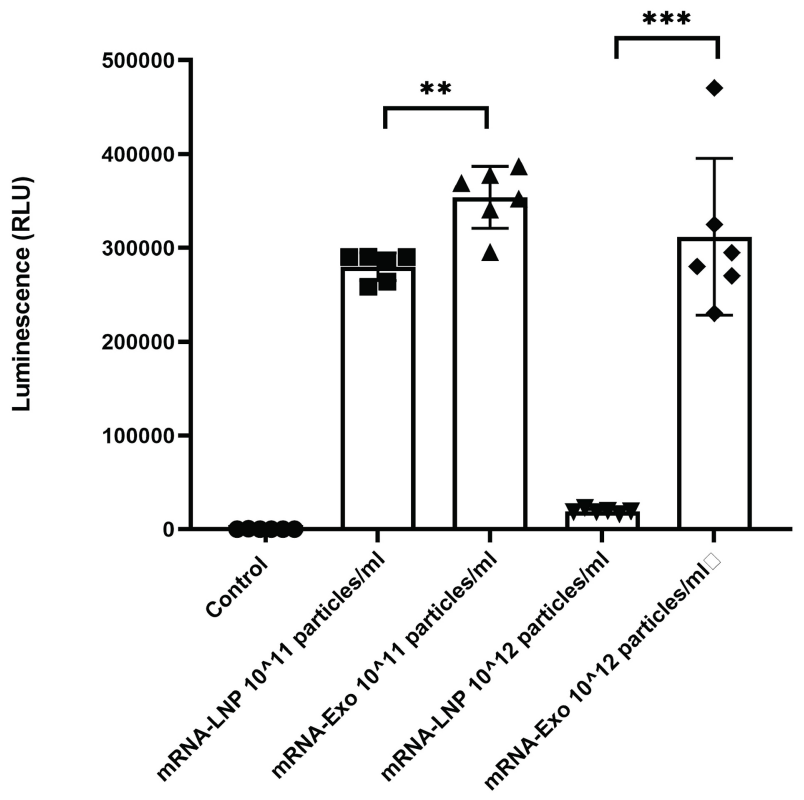

Figure 2 


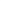


\begin{tabular}{l|l}
$\alpha$-SARS-CoV-2 & merge
\end{tabular}

븡

$\alpha-S A R S-C o V-2$

merge

merge

\section{Figure 5}




\section{$\mathrm{N}$-induced proliferation}
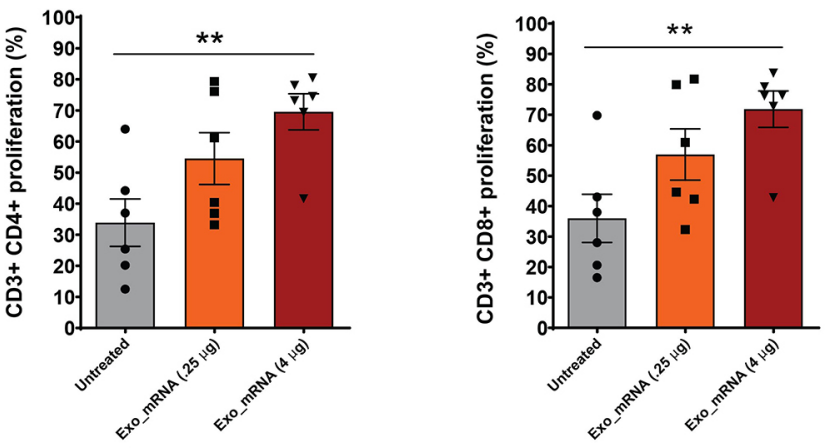

\section{S-induced proliferation}
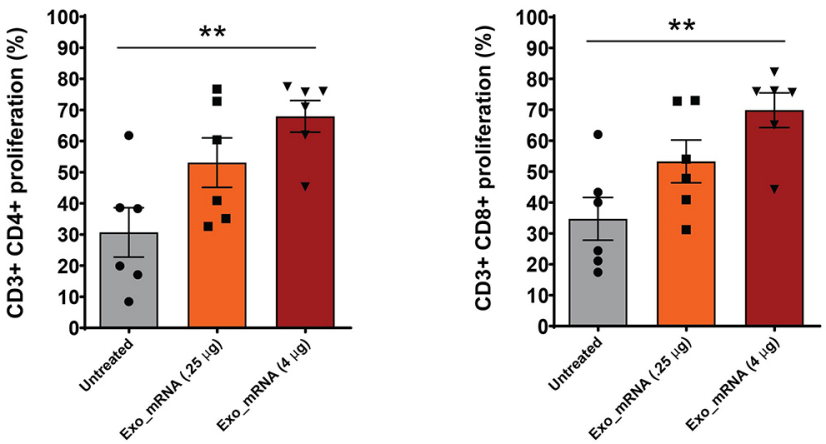


\section{$\mathrm{N}$-induced cytokine expression}
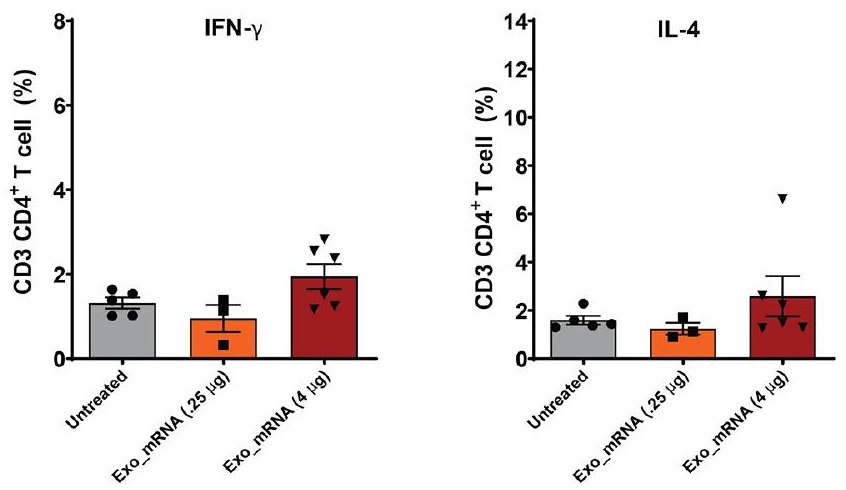

B S1-induced cytokine expression
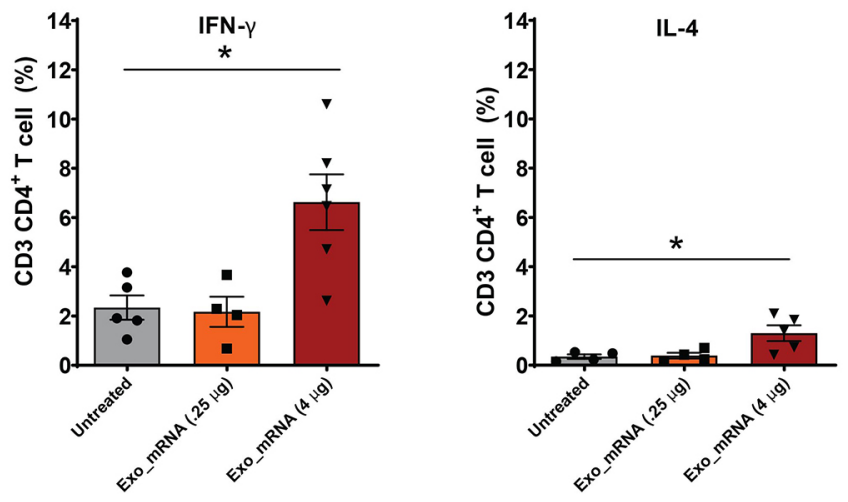


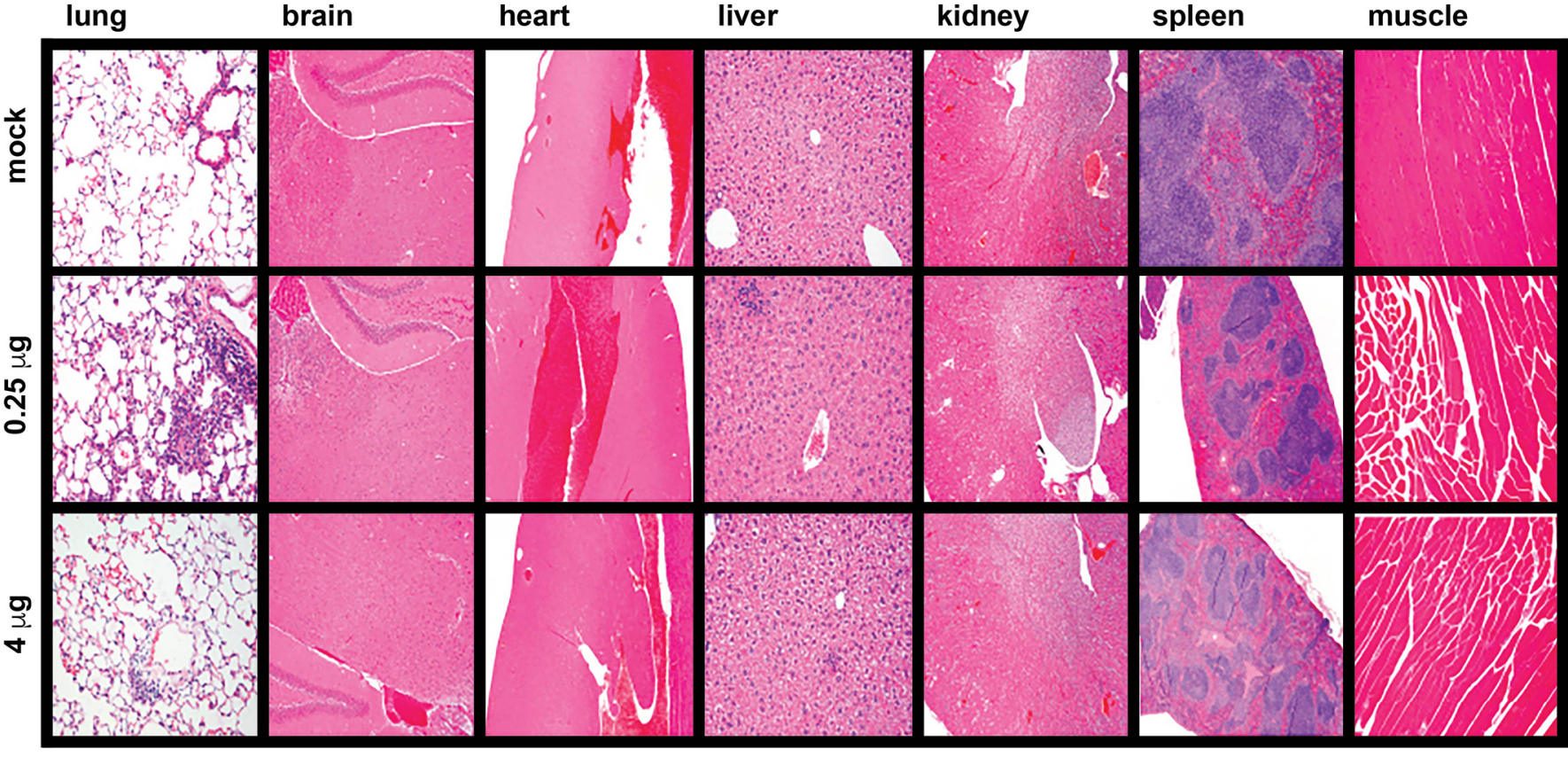

Figure 9 


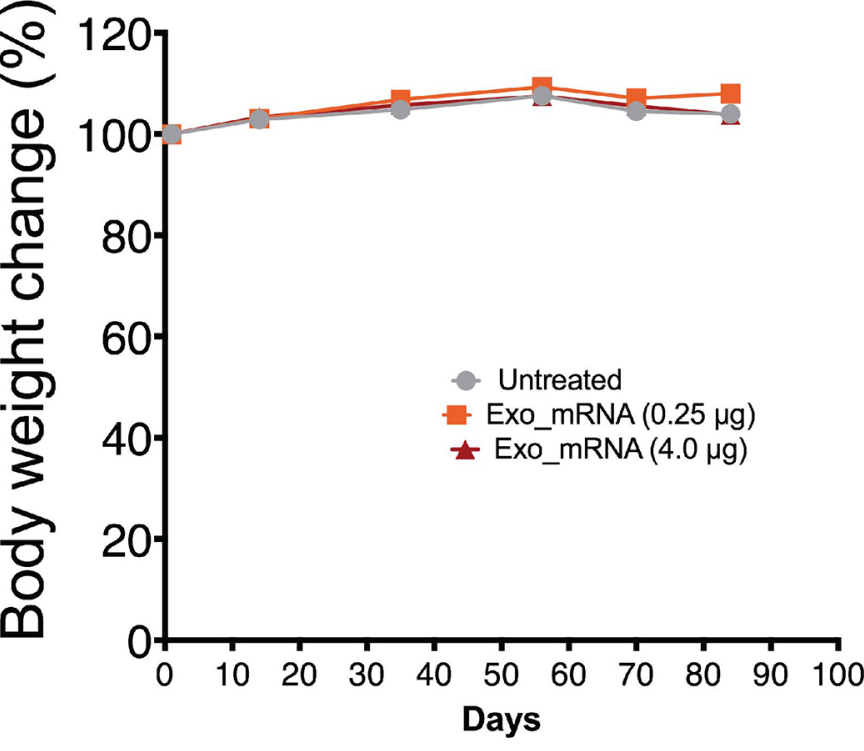

Supplemental Figure 1 
